\title{
Comportement d'interfaces metal-céramique réalisées par frittage et insertion à la coulée
}

\author{
N. Mesrati ${ }^{1}$, M. Courbière ${ }^{2}$, M. Laribi ${ }^{1}$, H. Yousfi ${ }^{1}$ et D. Treheux ${ }^{2}$ \\ ${ }^{1}$ Laboratoire Sciences et Génie des Matériaux, Département de Métallurgie, École \\ Nationale Polytechnique d'Alger, 10 avenue Hassen Badi El Harrach, Alger, Algérie \\ ${ }^{2}$ Laboratoire IFoS UMR 5621, Département STMS, École Centrale de Lyon, \\ 36 avenue Guy de Collongue, 69134 Ecully Cedex, France
}

\begin{abstract}
Mechanisms and Behavior of Metal-Ceramic interfaces processed by sintering and casting insertion: The development of ceramo-metallic systems, needs studies concerning the knowledge of the basic mechanisms responsible for optimal bonds. In addition other technological routes different from brazing or solid state bonding have to be studied.

The purpose of this work is to perform metal-ceramic assembling by sintering or casting insertion. Moreover, the reactions occuring during processing are identified.
\end{abstract}

\section{INTRODUCTION}

La technologie de l'assemblage des métaux aux céramiques a connu un développement tout d'abord largement empirique, sous forme de procédés conçus pour des conditions bien spécifiques [1-3] suivi par de nombreuses études fondamentales concernant les mécanismes d'adhésion [4-5], les contraintes résiduelles [6] ou la mécanique de la rupture interfaciale [7-8]. D'une façon générale, ces techniques d'assemblage peuvent se classer en deux catégories : celles qui font intervenir une phase liquide et celles se pratiquant entièrement à l'état solide. Pour analyser certains problèmes liés à de telles liaisons, nous avons repris cette distinction en élaborant des interfaces métal-céramique à l'état solide par frittage, et à l'état liquide, par insertion à la coulée de la céramique dans un moule métallique. Ce concept d'assemblage qui convient tout particulièrement à des pièces de formes complexes, exige cependant la maîtrise de l'influence de certains paramètres d'élaboration (Pression, température, atmosphère, état d'oxydation du métal, état des surfaces initiales etc...).

\section{MATERIAUX ET TECHNIQUES EXPERIMENTALES}

\subsection{Choix des matériaux}

Pour les besoins de nos expériences, nous avons retenu l'alumine $\left(\mathrm{Al}_{2} \mathrm{O}_{3}\right)$ qui est une céramique technique type oxyde très répandue. Cependant, la démarche suivie pourrait $s$ 'appliquer à des céramiques plus performantes $\left(\mathrm{ZrO}_{2}, \mathrm{SiC}, \mathrm{Si}_{3} \mathrm{~N}_{4}, \mathrm{SiALON}\right.$...) mais aussi plus onéreuses et plus délicates à mettre en oeuvre. Parmi les alumines disponibles, nous avons choisi la qualité $\mathrm{Al}_{23}$ de Degussa $\left(99,7 \% \mathrm{Al}_{2} \mathrm{O}_{3}\right.$ densité 3.7 à $3.95 \mathrm{~g} / \mathrm{cm}^{3}$, porosité fermée, taille des grains 10 à $60 \mu \mathrm{m}$, point de fusion $2030^{\circ} \mathrm{C}$ ).

L'alliage de moulage étudié est l'alpax (Al $12 \% \mathrm{Si}$ ) avec en comparaison l'aluminium pur (A5), ce qui a permis de mettre en évidence le rôle du Si. Les poudres métalliques utilisées pour le frittage $(\phi<10 \mu \mathrm{m})$ correspondent aux éléments utilisés dans les méthodes industrielles de liaisons céramique-métal (Mn) [3] ou dans la brasure des céramiques $(\mathrm{Cu})$ ou, enfin, qui sont classiques dans les alliages de construction thermomécanique ( $\mathrm{Fe}, \mathrm{Ni}$ et $\mathrm{Co}$ ). Par ailleurs, pour augmenter le mouillage du métal liquide sur la céramique, nous avons effectué des traitements de surface préalables de la céramique par slurry-coating (S.C) [9]. 
A partir de ces éléments, nous avons d'une part fritté les poudres métalliques autour de l'alumine pré frittée (massive) après compression unidirectionnelle à froid des poudres et, d'autre part, coulé l'alliage d'aluminium dans un moule métallique dans lequel la céramique a été insérée.

\subsection{Traitements de surface thermochimiques de la céramique}

Pour modifier les conditions de mouillabilité et d'accrochage entre les antagonistes, nous avons effectué, dans certains cas, des traitements de surface de l'alumine par Slurry-Coating "'S.C"' avant insertion à la coulée. Ce traitement, consiste à déposer les éléments d'addition sous forme de poudres liées par un vernis vinylique (suspension) à la surface de l'échantillon. Le traitement thermique qui suit est fait à $1000^{\circ} \mathrm{C}$ à l'air pendant des temps de traitement allant de 24 à $200 \mathrm{~h}$, ce qui permet de réaliser une zone d'interdiffusion de quelques dizaines de $\mu \mathrm{m}$. Nous avons utilisé les mêmes poudres que celles utilisées pour le frittage.

\section{CARACTERISATION}

Pour dégager les paramètres essentiels à prendre en compte afin de comprendre le comportement d'une interface métal/céramique, celle-ci a été caractérisée par diffraction $\mathrm{X}$ classique, par microscopie électronique à balayage avec microanalyse $\mathrm{X}$, et dans certains cas par microscopie électronique en transmission. Des essais de cisaillement ont été réalisés pour mesurer la résistance mécanique.

\section{RESULTATS}

\subsection{Caractérisation de la surface de l’alumine traitée par slurry-coating}

Des réactions interfaciales peuvent se produire entre un oxyde et le métal en particulier, si ce dernier présente un oxyde superficiel. C'est le cas du système $\mathrm{Cu}-\mathrm{Al}_{2} \mathrm{O}_{3}$ largement étudié par ailleurs [10-11].

Les résultats que nous avons obtenus par diffraction des rayons $\mathrm{X}$ à partir des poudres retenues et des traitements à l'air sont rassemblés dans le tableau 1. Lorsque le métal est oxydé (essais à l'air), l'adhérence est plus forte, voir importante (fer, cuivre, cobalt et manganèse) et la coloration de l'alumine montre une interdiffusion oxyde métallique/alumine. La phase principale formée par réaction avec l'alumine peut être une spinelle $\mathrm{CuAl}_{2} \mathrm{O}_{4}, \mathrm{CoAl}_{2} \mathrm{O}_{4}, \mathrm{NiAl}_{2} \mathrm{O}_{4}$ et $\mathrm{MnAl}_{2} \mathrm{O}_{4}$ ou un autre oxyde mixte $\mathrm{CuAlO}_{2}$ (cas du système $\mathrm{Cu}-\mathrm{Al}_{2} \mathrm{O}_{3}$ ). Dans notre cas, la présence du composé $\mathrm{CuAl}_{2} \mathrm{O}_{4}$ est caractérisée par une coloration brune de la surface de l'alumine et sa formation a lieu par interdiffusion des deux oxydes $\mathrm{Cu}_{2} \mathrm{O}$ et $\mathrm{Al}_{2} \mathrm{O}_{3}$ après oxydation de la poudre de cuivre en cuprite. Parfois, lorsque les structures sont compatibles une solution solide d'oxyde de type $\mathrm{M}_{2} \mathrm{O}_{3}\left(\mathrm{FeAl}_{2} \mathrm{O}_{3}\right)$ se forme à l'interface.

Pour plus de précisions et compte tenu de leur croissance rapide, nous avons analysé par microscopie électronique à transmission le spinelle cobalt $\mathrm{CoAl}_{2} \mathrm{O}_{4}$ et l'oxyde $\mathrm{CuAlO}_{2}$. Des lames minces ont été prélevées à la surface de l'alumine traitée par slurry-coating de $\mathrm{Co}$ et $\mathrm{Cu}$ respectivement. Les résultats obtenus mettent en évidence :

- pour le cobalt, la présence d'oxyde $\mathrm{CoAl}_{2} \mathrm{O}_{4}$ micro cristallisé $(0.3$ à $0.8 \mu \mathrm{m})$ (figure 1).

- pour le cuivre, l'analyse des défauts contenus dans l'oxyde $\mathrm{CuAlO}_{2}$ de structure rhomboedrique

décrite en hexagonal (figure 2), montre la présence de dislocations nombreuses et mobiles (figure 2a) ainsi que l'existence de macles de croissance (figure $2 b$ ) résultant d'un énantiomorphisme dû à la croissance de cet oxyde sur l'alumine. Ces oxydes mixtes ont donc des propriétés très particulières (microcristallisation pour $\mathrm{CoAl}_{2} \mathrm{O}_{4}$, dislocations très mobiles pour $\mathrm{CuAlO}_{2}$ ) pouvant avoir un rôle capital sur la tenue d'une interface les contenant. 
En outre, nous pouvons signaler ici que le traitement par Slurry-Coating utilisé permettrait à lui seul, du point de vue pratique, de réaliser un traitement superficiel thermochimique des céramiques conduisant à une modification de surface. Cette modification peut avoir un intérêt capital pour l'amélioration aussi bien des caractéristiques volumiques (résistance mécanique, $\mathrm{K}_{\mathrm{IC}}$, choc thermique) que surfaciques (frottement, usure).

Tableau 1. Composés formés et résiduels obtenus lors du dépôt du $\mathrm{Co}, \mathrm{Cu}, \mathrm{Ni}, \mathrm{Fe}$ et $\mathrm{Mn}$ par 'S.C'?

\begin{tabular}{|c|c|c|}
\hline Métal & $\begin{array}{c}\text { Produits de réaction } \\
\text { formés } \\
\text { en surface de } \mathrm{Al}_{2} \mathrm{O}_{3}\end{array}$ & $\begin{array}{c}\text { Composés } \\
\text { résiduels }\end{array}$ \\
\hline $\mathrm{Co}$ & $\mathrm{CoAl}_{2} \mathrm{O}_{4}$ & $\mathrm{CoO}$ \\
\hline $\mathrm{Cu}$ & $\begin{array}{c}\mathrm{CuAl} \mathrm{O}_{4} \\
\mathrm{CuAlO}_{2}\end{array}$ & $\begin{array}{c}\mathrm{CuO} \\
\mathrm{Cu}\end{array}$ \\
\hline $\mathrm{Ni}$ & $\mathrm{NiAl}_{2} \mathrm{O}_{4}$ & $\mathrm{NiO}$ \\
\hline $\mathrm{Fe}$ & $\mathrm{FeAl}_{2} \mathrm{O}_{4}$ & $\mathrm{Fe}_{2} \mathrm{O}_{3}$ \\
\hline $\mathrm{Mn}$ & $\mathrm{MnAl}_{2} \mathrm{O}_{4}$ & $\mathrm{MnO}$ \\
\hline
\end{tabular}

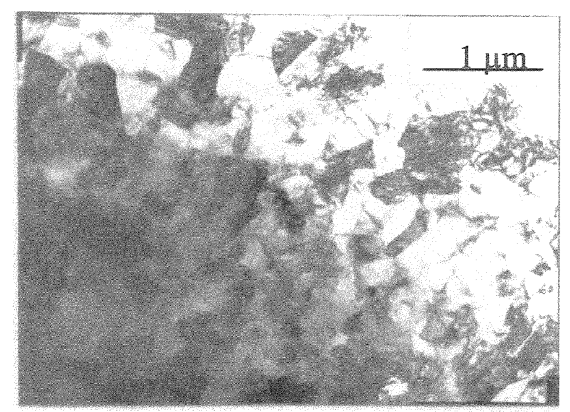

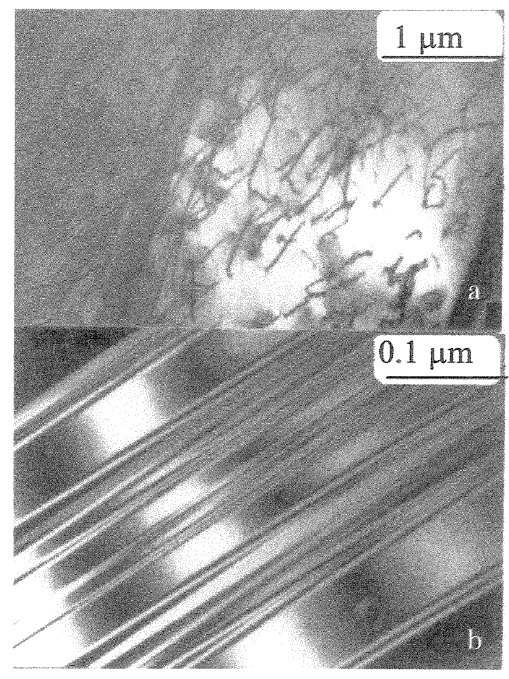

Figure 2. Phase $\mathrm{CuAlO}_{2}$ obtenue par "S.C" et observée en MET.

Figure 1. Phase $\mathrm{CoAl}_{2} \mathrm{O}_{4}$ obtenue par "S.C" et observée en MET.

\subsection{Analyse des systèmes Métal/Alumine obtenus par frittage}

Compte tenu des résultats obtenus par slurry-coating, nous avons retenu les couples $\mathrm{Fe} / \mathrm{Al}_{2} \mathrm{O}_{3} ; \mathrm{Ni} / \mathrm{Al}_{2} \mathrm{O}_{3}$; $\mathrm{Co} / \mathrm{Al}_{2} \mathrm{O}_{3}$ et $\mathrm{Cu} / \mathrm{Al}_{2} \mathrm{O}_{3}\left(\mathrm{Al}_{2} \mathrm{O}_{3}\right.$ non traitée) frittés à $1000^{\circ} \mathrm{C}$ pendant 4 heures sous vide primaire dynamique. Les échantillons sont ensuite traités à $1000^{\circ} \mathrm{C}$ sous $\mathrm{N}_{2} \mathrm{H}_{2}$.

\subsubsection{Traitements sous $\mathrm{N}_{2} \mathrm{H}_{2}$}

Comme précédemment, on note que la présence d'oxygène (éventuellement provenant d'une légère oxydation des poudres métalliques) peut suffire à favoriser la formation d'oxydes interfaciaux tel que $\mathrm{CoAl}_{2} \mathrm{O}_{4}$ par exemple (figure 3). Dans les mêmes conditions (sous balayage de $\mathrm{N}_{2} \mathrm{H}_{2}$ ), le couple $\mathrm{Fe}-\mathrm{Al}_{2} \mathrm{O}_{3}$ présente une interdiffusion Fe-Al. Par contre, le couple $\mathrm{Ni}_{-}-\mathrm{Al}_{2} \mathrm{O}_{3}$ ne présente aucune interdiffusion sensible à l'échelle micronique (figure 4). Ce qui est conforme aux études de Lourdin pour la température retenue ici [12]. 


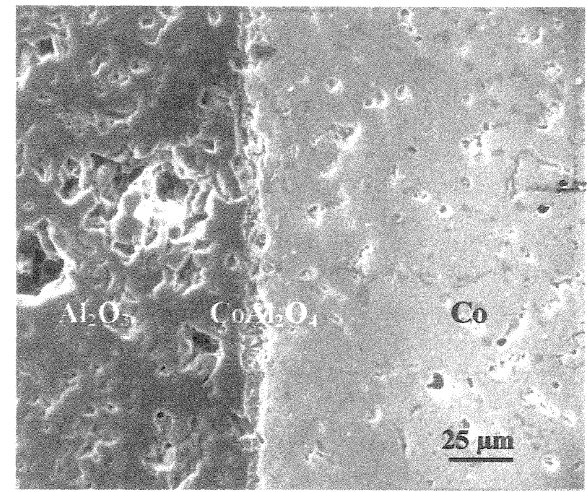

Figure 3. Zone interfaciale $\mathrm{Al}_{2} \mathrm{O}_{3}-\mathrm{Co}$ traité à $1000^{\circ} \mathrm{C}, \mathrm{t}=92 \mathrm{~h}$, sous $\mathrm{N}_{2} \mathrm{H}_{2}$

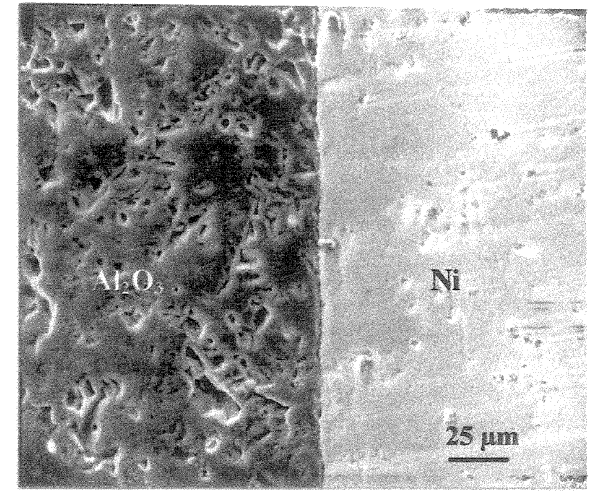

F igure 4. Zone interfaciale $\mathrm{Al}_{2} \mathrm{O}_{3}-\mathrm{Ni}$ traité à $1000^{\circ} \mathrm{C}, \mathrm{t}=92 \mathrm{~h}$, sous $\mathrm{N}_{2} \mathrm{H}_{2}$

\subsection{Analyse des systèmes AS12 coulé/Alumine}

Après coulée de l'alpax, l'examen de l'interface (figure 5), révèle une interface régulière entre l'alliage d'aluminium AS12 et l'alumine traitée par 'S.C' de $\mathrm{Cu}$ ainsi que la présence d'une zone de réaction entre l'oxyde de $\mathrm{Cu}$ et l'alumine. Les mêmes résultats sont obtenus dans le cas du système $\mathrm{AS} 12 / \mathrm{Co} / \mathrm{Al}_{2} \mathrm{O}_{3}$ alors que le système $\mathrm{Al} / \mathrm{Al}_{2} \mathrm{O}_{3}$ (non traité par "'S.C'), présente des défauts de mouillabilité.

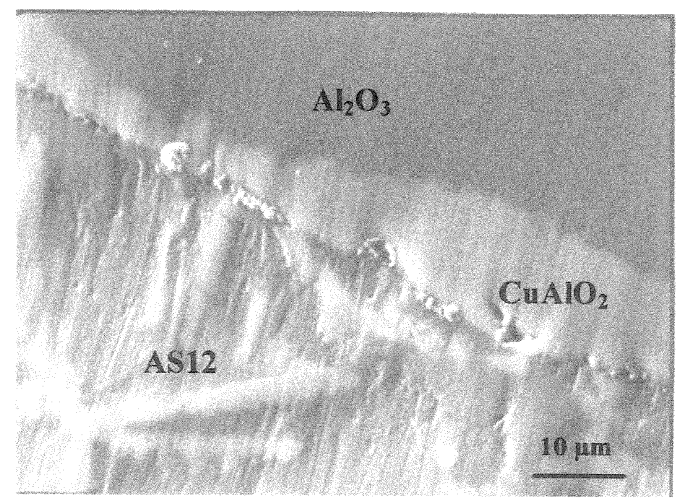

Figure 5. Zone interfaciale $\mathrm{AS} 12-\mathrm{Cu}-\mathrm{Al}_{2} \mathrm{O}_{3}$, traité à $1000^{\circ} \mathrm{C}, \mathrm{t}=48 \mathrm{~h}$, à l'air.

\subsubsection{Analyse mécanique}

Les essais de cisaillement nous montrent que la rupture, dans le cas des systèmes à base d'alpax comme alliage de coulée, se fait sous une contrainte plus importante que pour ceux à base de l'aluminium A5 (figure 6). On constate l'effet bénéfique du Si qui est donc favorable à l'adhérence dans les systèmes étudiés. Un post-traitement du couple $\mathrm{AS} 12 / \mathrm{Al}_{2} \mathrm{O}_{3}$, effectué à $500^{\circ} \mathrm{C}$ pendant 48 heures, ne favorise pas la bonne tenue au cisaillement de l'interface. Le prétraitement par Slurry-Coating de l'alumine est bénéfique dans le cas du cuivre mais moins efficace pour le cobalt. 


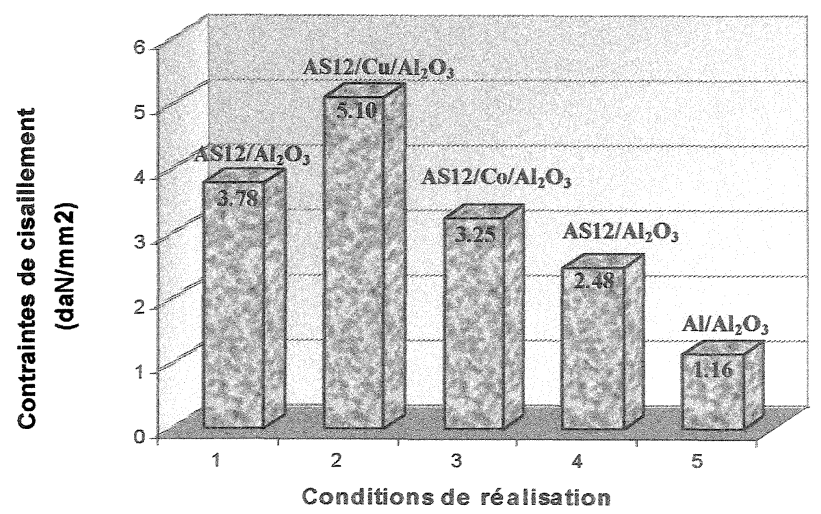

\begin{tabular}{|c|c|}
\hline 1 & 2 \\
\hline Préch & "S.C.C".Cu \\
\hline $\begin{array}{l}\mathrm{Al}_{2} \mathrm{O}_{3} \\
\mathrm{~T}_{1}^{\circ}=248^{\circ} \mathrm{C} \\
\operatorname{tps}_{1}=2 \mathrm{~h}\end{array}$ & $\begin{array}{l}\mathrm{T}_{2}{ }^{\circ}=955^{\circ} \mathrm{C} \\
\operatorname{tps}_{2}=25 \mathrm{~h}\end{array}$ \\
\hline 3 & 4 \\
\hline "'S.C"'.Co & Préch \\
\hline $\mathrm{T}_{3}{ }^{\circ}=955^{\circ} \mathrm{C}$ & $\mathrm{Al}_{2} \mathrm{O}_{3}$ \\
\hline $\mathrm{Tps}_{3}=25 \mathrm{~h}$ & $\begin{array}{l}\mathrm{T}_{4}{ }^{\circ}=283^{\circ} \mathrm{C} \\
\mathrm{Tps}_{4}=2 \mathrm{~h}\end{array}$ \\
\hline 5 & Recuit/AS12 \\
\hline Préch & $\mathrm{T}_{4^{\circ}}{ }^{\circ}=500^{\circ} \mathrm{C}$ \\
\hline $\begin{array}{l}\mathrm{T}_{5}^{\circ}=313^{\circ} \mathrm{C} \\
\operatorname{tps}_{5}=2 \mathrm{~h}\end{array}$ & $\mathrm{tps}_{4}=24 \mathrm{~h}$ \\
\hline
\end{tabular}

Figure 6. Résistance de l'interface en cisaillement en fonction des conditions d'élaboration des multimatériaux étudiés. Températures et temps $\left(\mathrm{T}^{\circ}, \mathrm{tps}\right)$ du préchauffage, du Slurry-Coating et du recuit de l'alpax (AS12).

\section{CONCLUSION}

Parmi les traitements de surface de céramiques que nous avons mis au point, ceux à base du Co et surtout $\mathrm{du} \mathrm{Cu}$, semblent conduire aux meilleurs résultats d'accrochage suivant notre mode de caractérisation. Lors de la coulée dans le moule métallique, les oxydes ternaires formés par les divers systèmes élaborés sont responsables des bons résultats obtenus dans le cas du couple alpax/métal/alumine comme le montrent les essais de cisaillement. Apparemment, le silicium est favorable quant à l'amélioration de la liaison entre les deux constituants principaux, sachant que les contraintes de cisaillement relatives aux couples $\mathrm{AS} 12 / \mathrm{Al}_{2} \mathrm{O}_{3}$, sont plus importantes que celles obtenues pour les couples $\mathrm{Al} / \mathrm{Al}_{2} \mathrm{O}_{3}$.

L'adhésion semble pouvoir se résoudre théoriquement, par la mise au point de systèmes réactifs ainsi que par l'analyse des éléments d'addition favorables et pratiquement par une conception géométrique capable d'accommoder les différentes contraintes surtout thermiques.

Nous confirmons que l'adhérence est fortement dépendante des réactions interfaciales pouvant se produire à l'interface métal-céramique et des propriétés mécaniques et thermiques des phases formées.

Remerciements: Nous tenons à remercier C. ESNOUF et C. BERAUD pour la réalisation des observations en MET.

\section{Références}

[1] W. H. Kohl, Handbook of Materials and Techniques for Vacuum Devices, Reinhold Publ. Corp, New York (1967).

[2] A. Roth, Vacuum Sealing Techniques, Pergamon Press, Oxford (1966).

[3] M. Courbière, D. Tréheux, C. Beraud, C. Esnouf, Liaisons métal-céramique : techniques et aspects physicochimiques, Ann. Chim. Sci. Mat. 12 (1987) 295-312.

[4] Y. Naidich, $9^{\text {th }}$ Cimtec Ceramics Getting into 2000's Partc, P. Vincenzini (Ed) Techna srl (1999) 965-978.

[5] D. Tréheux, P. Lourdin, V. Guipont and D. Juvé, Metal Ceramic solid state bonding mechanisms and 
mechanics Scripta Metall et Mater 31(8) (1994) 1055-1060.

[6] D. Tréheux, S. Fayeulle, V. Guipont, J. P. Jacquemin, Interfacial Science in Ceramic Joining, A. Bellosi et al. (Eds) Kluwer Akademic Publishers (1998) 311-318.

[7] A. G. Evans, J.W. Hutchinson, Acta Met 37 (1989) 909.

[8] A. G. Evans, M. Ruhle, B. J. Dalghlish and P. G. Chatalambides, Mat. Sci. Eng. A126 (1990) 53-64

[9] N. Mesrati, D. Nguyen, D. Tréheux, Revue de Métallurgie-CIT/Science et Génie des matériaux, 12 (1993) 1673-1680.

[10] A. Kara-slimane, B. Mbongo and D. Treheux, J. Adhesion Sci. Technol, 13 (1) (1999) 35-48.

[11] S. K. MISRA, A. C. D. CHAKLADER, J. Am. Ceram. Soc. 40 (10) (1963) 509.

[12] P. Lourdin, Thèse de Doctorat, Ecole Centrale de Lyon, Génie des Matériaux N 92-31 (1992). 